\title{
Adaptive Cooperative Manipulation with Rolling Contacts
}

\author{
Christos K. Verginis, Wenceslao Shaw Cortez, and Dimos V. Dimarogonas
}

\begin{abstract}
In this paper we present a novel adaptive cooperative manipulation controller for multiple mobile robots with rolling contacts. Whereas conventional cooperative manipulation methods require rigid contact points, our approach exploits rolling effects of passive end-effectors and does not require force/torque sensing. The removal of rigidity allows for more modular grasping, increased application to more object types, and online adjustment of the grasp. The proposed approach is robust to uncertain dynamics of the object and agents including object center of mass, inertia, weight, and Coriolis terms. Furthermore, we present a novel closed-form internal force controller that guarantees no slip throughout the manipulation task. Also, the design of the adaptive controller ensures boundedness of the estimated model parameters in predefined sets. Numerical simulations validate the effectiveness of the proposed approach.
\end{abstract}

\section{INTRODUCTION}

Recent technological advancements have led to the concept of automated manufacturing where the ability to transport objects/packages autonomously is key to the production process. One popular, modular approach to perform object transport is via cooperative manipulation, which entails the transport/manipulation of an object by using multiple mobile manipulators. Due to the different objects that must be transported in such a setting, the cooperative manipulation methods should be robust to uncertainties in object weight, inertia, shape, and even center of mass location.

Existing methods in cooperative manipulation aim to track a desired object reference trajectory using robotic manipulators on mobile bases. Multiple robots allow for carrying heavy loads and executing dexterous maneuvers. Early works in cooperative manipulation focused on hybrid force/position and impedance control schemes [1], [2]. Other approaches focused on decentralization of the agents [3] and adaptive controllers [4], [5]. However, those methods rely on the assumption that each agent is rigidly fixed to the object, allowing it to apply any force/torque at the contact point. The rigidity assumption is highly restrictive as it only applies to objects on which a rigid grasp can be formed, excluding, e.g., objects with smooth surfaces or large boxes/spheres (e.g packages), which cannot be rigidly grasped by a simple gripper. Furthermore, many existing approaches are dependent on force/torque sensors mounted on each robot, which can

The authors are with the School of Electrical Engineering, KTH Royal Institute of Technology, SE-100 44, Sweden (Email: cverginis, wencsc, dimosekth.se). This work was supported by the H2020 ERC Starting Grant BUCOPHSYS, the European Union's Horizon 2020 Research and Innovation Programme under the GA No. 731869 (Co4Robots), the Swedish Research Council (VR), the Knut och Alice Wallenberg Foundation (KAW) and the Swedish Foundation for Strategic Research (SSF). be expensive or difficult to equip appropriately on a fleet of mobile manipulators.

In this work we aim to remove the rigidity assumption that is characteristic of current cooperative manipulation approaches, and relax the dependency on tactile sensors to contact location sensors only. We propose exploiting natural rolling of a passive end-effector to accomplish the cooperative manipulation objective. Non-rigid/rolling contacts increase the number of objects that can be grasped, increase the workspace of the system, and allow for modular manipulation scenarios in which robots can be swapped in/out to adjust the grasp online. Note that by employing rolling contacts, the cooperative manipulation problem here is similar to robotic grasping [6] albeit with moving "fingers."

Despite advancements in the literature, existing methods from cooperative manipulation and robotic grasping are not applicable to the cooperative manipulation problem posed here. The aforementioned works in cooperative manipulation [1]-[5] are not applicable due to the dependency on rigid contacts. Rolling contacts complicate the problem as each contact may only apply a force that respects friction cone constraints to prevent slip, instead of an arbitrary wrench associated with rigid contacts [7]. Early robotic grasping approaches required exact knowledge of the agent's dynamics [7], [8]. Other recent techniques are robust to model uncertainties, but neglect rolling effects or dynamics [9][11], while other more sensor-deprived approaches assume the object is weightless [12], [13]. The approach from [14] assumes a priori bounded states, which does not apply to the mobile manipulators considered here. Adaptive control schemes that have also been developed require force and contact location sensing, and assume boundedness of the uncertain parameter estimates [15], [16], or are limited to set-point (constant reference) manipulation [17]. Thus, there is no robust cooperative manipulation approach that ensures stability to a reference trajectory with non-rigid, rolling contacts and no force/torque sensing. Furthermore, for the collaborative manipulation proposed here, it is critical to ensure the object does not slip. This is neglected by most of the aforementioned approaches, which assume either rigid grasps or simply no slip without guarantees. Methods of ensuring slip prevention are developed typically by solving an optimization problem online [9], [18], [19]. However [9], [19] neglect the dynamics of the system, which may perturb the system and cause slip. The approach in [18] uses a conservative bound on the dynamics, which overcompensates the amount of force required to hold the object. Finally, most related works (e.g., [1], [4], [5], [8], [13]) consider accurate knowledge of the object center of mass, which 
can be difficult to obtain in practice, especially in cases of complicated object shapes.

In this paper, we present a novel adaptive cooperative manipulation control scheme for rolling contact points that does not use force/torque sensing. The proposed controller ensures asymptotic stability to a reference trajectory and is robust to uncertain dynamic model parameters including object/robot center of mass, inertia, and weight. Furthermore, we propose a novel internal force controller that ensures no slip during the manipulation motion without neglecting nor overcompensating for the system dynamics. Numerical simulations are used to validate the proposed approach.

The rest of the paper is organized as follows. Section II describes the problem considered here. Section III introduces the proposed control scheme, and a simulation example is given in Section IV Section V concludes the paper.

Notation: Throughout this paper, the notation $\boldsymbol{v}^{\mathcal{E}}$ indicates that the vector $\boldsymbol{v}$ is written with respect to a frame $\mathcal{E}$, and if there is no explicit frame defined, $v$ is written with respect to the inertial frame, $\mathcal{P}$. The operator $(\cdot) \times$ denotes the skewsymmetric matrix representation of the cross-product. $S O(3)$ denotes the special orthogonal group of dimension 3 , and $S^{n}$ is the unit $(n-1)$-dimensional sphere. The $r \times r$ identity matrix is denoted by $I_{r}$ and the $r$-dimensional vector of zeros by $\mathbf{0}_{r}$. The terms $\succeq, \preceq$ denote element-wise vector inequalities. The null space of a matrix $B$ is denoted $\mathfrak{N}(B)$, and the interior of a set $\mathscr{A}$ is $\operatorname{Int}(\mathscr{A})$.

\section{PROBLEM FORMULATION}

Consider $N \in \mathbb{N}$ robotic agents, consisting of a holonomic moving base and a robotic arm, grasping a rigid object in $3 \mathrm{D}$ space. Let their generalized joint space variables and respective derivatives be $\boldsymbol{q}_{i}, \dot{\boldsymbol{q}}_{i} \in \mathbb{R}^{n_{i}}$ with $n_{i} \geq 3, \forall i \in$ $\mathscr{N}:=\{1, \ldots, N\}$. Here $\boldsymbol{q}_{i}$ consists of the degrees of freedom of the robotic arm as well as the moving base. The overall joint configuration is then $\boldsymbol{q}:=\left[\boldsymbol{q}_{1}^{T}, \ldots, \boldsymbol{q}_{N}^{T}\right]^{T}$, $\dot{\boldsymbol{q}}:=\left[\dot{\boldsymbol{q}}_{1}^{T}, \ldots, \dot{\boldsymbol{q}}_{N}^{T}\right]^{T} \in \mathbb{R}^{n}$ with $n:=\sum_{i \in \mathscr{N}} n_{i}$. Each agent has a smooth, convex "fingertip" (i.e. passive end-effector) of high stiffness that is in contact with an object via a smooth contact surface. Let the inertial frame be denoted by $\mathcal{P}$, and a fingertip frame, $\mathcal{F}_{i}$, fixed at the point $\boldsymbol{p}_{f_{i}} \in \mathbb{R}^{3}$ on each agent end-effector. The translational and rotational velocities of $\mathcal{F}_{i}$ with respect to $\mathcal{P}$ are denoted by $\boldsymbol{v}_{f_{i}}, \boldsymbol{\omega}_{f_{i}} \in \mathbb{R}^{3}$, respectively. The rotation matrix from $\mathcal{F}_{i}$ to $\mathcal{P}$ is $R_{p f_{i}}:=R_{p f_{i}}\left(\boldsymbol{q}_{i}\right) \in$ $S O(3)$. The contact frame, $\mathcal{C}_{i}$, is located at the contact point, $\boldsymbol{p}_{c_{i}} \in \mathbb{R}^{3}$ and defined as a Gauss frame [20] where one of the axes is defined orthonormal to the contact plane. The vector from $\mathcal{F}_{i}$ to $\mathcal{C}_{i}$ is $\boldsymbol{p}_{f c_{i}}:=\boldsymbol{p}_{c_{i}}-\boldsymbol{p}_{f_{i}} \in \mathbb{R}^{3}$. A visual representation of the contact geometry for the $i$ th agent is shown in Figure 1 .

The dynamics of agent $i$ in the grasp is defined by [20]:

$$
M_{i} \ddot{\boldsymbol{q}}_{i}+C_{i} \dot{\boldsymbol{q}}_{i}+\boldsymbol{g}_{i}=-J_{h_{i}}^{T} \boldsymbol{f}_{c_{i}}+\boldsymbol{u}_{i}
$$

where $M_{i}:=M_{i}\left(\boldsymbol{q}_{i}\right) \in \mathbb{R}^{n_{i} \times n_{i}}$ is the positive definite inertia matrix, $C_{i}:=C_{i}\left(\boldsymbol{q}_{i}, \dot{\boldsymbol{q}}_{i}\right) \in \mathbb{R}^{n_{i} \times n_{i}}$ is the Coriolis/centrifugal matrix, $\boldsymbol{g}_{i}:=\boldsymbol{g}_{i}\left(\boldsymbol{q}_{i}\right)$ is the gravity torque, $\boldsymbol{f}_{c_{i}} \in \mathbb{R}^{3}$ is the contact force, $\boldsymbol{u}_{i} \in \mathbb{R}^{n_{i}}$ is the joint torque control input, and

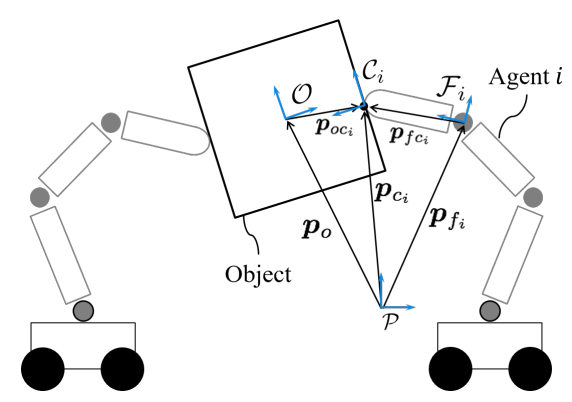

Fig. 1: Two robotic agents in contact with an object.

$J_{h_{i}}:=J_{h_{i}}\left(\boldsymbol{q}_{i}, \boldsymbol{p}_{f c_{i}}\right) \in \mathbb{R}^{3 \times n_{i}}$ is the agent Jacobian matrix, defined by

$$
J_{h_{i}}\left(\boldsymbol{q}_{i}, \boldsymbol{p}_{f c_{i}}\right):=\left[\begin{array}{ll}
I_{3} & -\left(\boldsymbol{p}_{f c_{i}}\right) \times
\end{array}\right] J_{s_{i}}\left(\boldsymbol{q}_{i}\right),
$$

where $J_{s_{i}}\left(\boldsymbol{q}_{i}\right) \in \mathbb{R}^{6 \times n_{i}}$ is the manipulator Jacobian that maps $\dot{\boldsymbol{q}}_{i} \mapsto\left(\boldsymbol{v}_{f_{i}}, \boldsymbol{\omega}_{f_{i}}\right)$ [20]. The full hand Jacobian matrix is $J_{h}:=\operatorname{diag}\left\{\left[J_{h_{i}}\right]_{i \in \mathscr{N}}\right\} \in \mathbb{R}^{3 N \times n}$. We emphasize that the dynamical parameters (masses, moments of inertia) appearing in the terms $M_{i}, C_{i}, \boldsymbol{g}_{i}$ are considered to be unknown, $\forall i \in \mathscr{N}$. The dynamics (1) can be written in vector form as

$$
M \ddot{\boldsymbol{q}}+C \dot{\boldsymbol{q}}+\boldsymbol{g}=-J_{h}^{T} \boldsymbol{f}_{c}+\boldsymbol{u}
$$

where $M:=M(\boldsymbol{q}):=\operatorname{diag}\left\{\left[M_{i}\right]_{i \in \mathscr{N}}\right\}, C:=C(\boldsymbol{q}, \dot{\boldsymbol{q}}):=$ $\operatorname{diag}\left\{\left[C_{i}\right]_{i \in \mathscr{N}}\right\} \in \mathbb{R}^{n \times n}$, and $\boldsymbol{g}:=\boldsymbol{g}(\boldsymbol{q}):=\left[\boldsymbol{g}_{1}^{T}, \ldots, \boldsymbol{g}_{N}^{T}\right]^{T}$, $\boldsymbol{f}_{c}:=\left[\boldsymbol{f}_{c_{1}}^{T}, \ldots, \boldsymbol{f}_{c_{N}}^{T}\right]^{T}, \boldsymbol{u}:=\left[\boldsymbol{u}_{1}^{T}, \ldots, \boldsymbol{u}_{N}^{T}\right]^{T} \in \mathbb{R}^{n}$.

A common assumption in the majority of the related literature is that the object center of mass is accurately known, which is typically not the case in practice. In this work, we assume tracking of a traceable point $\boldsymbol{p}_{o}$ on the object surface instead of the center of mass, whose information is considered unknown. Note that appropriate sensor equipment, e.g., cameras and markers, can accurately track such points in practice. Hence, to remove the dependency on an unknown object center of mass, we perform a standard rigid body transformation to the conventional object dynamics as follows. Let $\mathcal{O}$ be a reference frame fixed at $\boldsymbol{p}_{o}$, which is not coincident with the object center of mass. Let also $R_{p o} \in S O(3)$ be the respective rotation matrix, which maps from $\mathcal{O}$ to $\mathcal{P}$. Let $\boldsymbol{x}_{o}:=\left[\boldsymbol{p}_{o}^{T}, \boldsymbol{\eta}_{o}^{T}\right]^{T} \in \mathbb{M}:=\mathbb{R}^{3} \times \mathbb{T}$, $\boldsymbol{v}_{o}:=\left[\dot{\boldsymbol{p}}_{o}^{T}, \boldsymbol{\omega}_{o}^{T}\right]^{T} \in \mathbb{R}^{6}$ denote the pose and generalized velocity of the object frame, with $\boldsymbol{\eta}_{o} \in \mathbb{T}$ an orientation vector and $\mathbb{T}$ the associated domain. The position vector from $\mathcal{O}$ to the respective contact point is $\boldsymbol{p}_{o c_{i}}:=\boldsymbol{p}_{c_{i}}-\boldsymbol{p}_{o} \in \mathbb{R}^{3}$.

We denote the actual object center of mass location with $\overline{\boldsymbol{p}}_{o} \in \mathbb{R}^{3}$, and without loss of generality we align the object body frame with $\mathcal{O}$ so that $\boldsymbol{\eta}_{o}=\overline{\boldsymbol{\eta}}_{o}$ and $\overline{\boldsymbol{\omega}}_{o}=\boldsymbol{\omega}_{o}$. Thus we let $\overline{\boldsymbol{x}}_{o}:=\left[\overline{\boldsymbol{p}}_{o}^{T}, \boldsymbol{\eta}_{o}^{T}\right]^{T} \in \mathbb{M}$ be the object pose with respect to the inertial frame $\mathcal{P}$ and $\overline{\boldsymbol{v}}_{o}:=\left[\dot{\overline{\boldsymbol{p}}}_{o}^{T}, \boldsymbol{\omega}_{o}^{T}\right]^{T} \in \mathbb{R}^{6}$. Let $\overline{\boldsymbol{p}}_{o c_{i}}:=\boldsymbol{p}_{c_{i}}-\overline{\boldsymbol{p}}_{o}$ with $\boldsymbol{p}_{o c}:=\left[\boldsymbol{p}_{o c_{1}}^{T}, \ldots, \boldsymbol{p}_{o c_{N}}^{T}\right]^{T} \in \mathbb{R}^{3 N}$. The conventional object dynamics with respect to the object center of mass are given by the Newton-Euler formulation:

$$
\bar{M}_{o} \dot{\overline{\boldsymbol{v}}}_{o}+\bar{C}_{o} \overline{\boldsymbol{v}}_{o}+\overline{\boldsymbol{g}}_{o}=\bar{G} \boldsymbol{f}_{c}
$$

where $\bar{M}_{o}:=\bar{M}_{o}\left(\overline{\boldsymbol{\eta}}_{o}\right) \in \mathbb{R}^{6 \times 6}$ is the object inertia matrix, $\bar{C}_{o}:=\bar{C}_{o}\left(\overline{\boldsymbol{\eta}}_{o}, \overline{\boldsymbol{\omega}}_{o}\right) \in \mathbb{R}^{6 \times 6}$ is the object Coriolis and 
centrifugal matrices, $\bar{G}:=\bar{G}\left(\overline{\boldsymbol{p}}_{o c}\right) \in \mathbb{R}^{6 \times 3 N}$ is the grasp map, and $\overline{\boldsymbol{g}}_{o} \in \mathbb{R}^{6}$ is the gravity acting on the object.

The grasp map, $\bar{G}$, maps the concatenated contact force, $f_{c} \in \mathbb{R}^{3 N}$, to the net wrench acting on the object center of mass and is defined by $\bar{G}:=\left[\bar{G}_{1}, \ldots, \bar{G}_{N}\right]$ where $\bar{G}_{i}:=$ $\bar{G}_{i}\left(\overline{\boldsymbol{p}}_{o c_{i}}\right):=\left[I_{3},-\left(\overline{\boldsymbol{p}}_{o c_{i}}\right) \times\right]^{\top} \in \mathbb{R}^{6 \times 3}$. We note that $\bar{G}$ is the conventional grasp map commonly used in grasping [20].

To perform the rigid body transformation let $J_{a}:=J_{a}\left(\overline{\boldsymbol{\eta}}_{o}\right) \in \mathbb{R}^{6 \times 6}$ be defined as:

$$
J_{a}\left(\overline{\boldsymbol{\eta}}_{o}\right):=\left[\begin{array}{cc}
I_{3} & \left(R_{p o} \boldsymbol{p}_{\bar{O}}^{\mathcal{O}}\right) \times \\
\mathbf{0}_{3} & I_{3}
\end{array}\right]
$$

where $\boldsymbol{p}_{\bar{O} O}^{\mathcal{O}}:=\boldsymbol{p}_{o}^{\mathcal{O}}-\overline{\boldsymbol{p}}_{o}^{\mathcal{O}}$, such that $\overline{\boldsymbol{v}}_{o}$ and $\boldsymbol{v}_{o}$ can be related via $\overline{\boldsymbol{v}}_{o}=J_{a} \boldsymbol{v}_{o}$, which is derived by differentiating $\overline{\boldsymbol{p}}_{o}=$ $\boldsymbol{p}_{o}+R_{p o}\left(\overline{\boldsymbol{p}}_{o}^{\mathcal{O}}-\boldsymbol{p}_{o}^{\mathcal{O}}\right)$. Note that $\boldsymbol{p}_{\bar{o} O}^{\mathcal{O}}$ is constant.

Substitution of $\overline{\boldsymbol{v}}_{o}=J_{a} \boldsymbol{v}_{o}$ and left multiplication by $J_{a}^{T}$ in (3) yields the adjusted object dynamics with respect to $\boldsymbol{p}_{o}$ :

$$
M_{o} \dot{\boldsymbol{v}}_{o}+C_{o} \boldsymbol{v}_{o}+\boldsymbol{g}_{o}=G \boldsymbol{f}_{c},
$$

where $M_{o}:=M_{o}\left(\overline{\boldsymbol{\eta}}_{o}\right):=J_{a}^{T} \bar{M}_{o} J_{a} \in \mathbb{R}^{6 \times 6}, C_{o}:=$ $C_{o}\left(\overline{\boldsymbol{\eta}}_{o}, \boldsymbol{\omega}_{o}\right):=J_{a}^{T}\left(\bar{M}_{o} \dot{J}_{a}+\bar{C}_{o} J_{a}\right) \in \mathbb{R}^{6 \times 6}, \boldsymbol{g}_{o}:=\boldsymbol{g}_{o}\left(\overline{\boldsymbol{\eta}}_{o}\right):=$ $J_{a}^{T} \overline{\boldsymbol{g}}_{o} \in \mathbb{R}^{6}$, and $G:=J_{a}^{T} \bar{G} \in \mathbb{R}^{6 \times 6}$, for which it holds $G=\left[G_{1}, \ldots, G_{N}\right]$, with

$$
G_{i}=J_{a}^{T} \bar{G}_{i}=\left[\begin{array}{c}
I_{3} \\
-\left(R_{p o} \boldsymbol{p}_{\bar{o} o}^{\mathcal{O}}\right) \times+\left(\overline{\boldsymbol{p}}_{o c_{i}}\right) \times
\end{array}\right]=\left[\begin{array}{c}
I_{3} \\
\left(\boldsymbol{p}_{o c_{i}}\right) \times
\end{array}\right],
$$

where we have used $R_{p o} \overline{\boldsymbol{p}}_{\bar{o} o}^{\mathcal{O}}=\boldsymbol{p}_{\bar{o} o}$. Note that $G=G\left(\boldsymbol{p}_{o c}\right)$, i.e., $G$ is not dependent on $\overline{\boldsymbol{p}}_{o}$. Note also by the relation $\overline{\boldsymbol{p}}_{o}=\boldsymbol{p}_{o}-R_{p o} \boldsymbol{p}_{\bar{o} o}^{\mathcal{O}}$, that $M_{o}, C_{o}, \boldsymbol{g}_{o}$ are functions of $\overline{\boldsymbol{\eta}}_{o}=\boldsymbol{\eta}_{o}$, $\overline{\boldsymbol{\omega}}_{o}=\boldsymbol{\omega}_{o}$ with dependency on the constant but unknown term $\boldsymbol{p}_{\overline{\mathcal{O}} \mathrm{O}}$. We also note the following relation that will be needed subsequently:

$$
\bar{G}_{i}^{T} \overline{\boldsymbol{v}}_{o}=\left[\begin{array}{c}
I_{3} \\
\left(\boldsymbol{p}_{c_{i}}-\overline{\boldsymbol{p}}_{o}\right) \times
\end{array}\right]^{T}\left[\begin{array}{cc}
I_{3} & \left(R_{p o} \boldsymbol{p}_{\bar{O} o}^{\mathcal{O}}\right) \times \\
\mathbf{0}_{3} & I_{3}
\end{array}\right] \boldsymbol{v}_{o}=G_{i}^{T} \boldsymbol{v}_{o}
$$

Similarly to the agents, the object dynamic parameters appearing in the terms $M_{o}, C_{o}, \boldsymbol{g}_{o}$ are considered to be unknown.

Regarding the object orientation, we use the unit quaternion choice $\boldsymbol{\eta}_{o}:=\left[\phi_{o}, \boldsymbol{\epsilon}_{o}^{T}\right]^{T} \in \mathbb{T}:=\mathrm{S}^{3}$, where $\phi_{o} \in[-1,1]$ and $\epsilon_{o} \in \mathbb{R}^{3}$ are the scalar and vector part, respectively, satisfying $\phi_{o}^{2}+\boldsymbol{\epsilon}_{o}^{T} \boldsymbol{\epsilon}_{o}=1$. Moreover, it holds that [5]

$$
\dot{\boldsymbol{\eta}}_{o}=\frac{1}{2} E_{\eta}\left(\boldsymbol{\eta}_{o}\right) \boldsymbol{\omega}_{o} \Rightarrow \boldsymbol{\omega}_{o}=2 E_{\eta}\left(\boldsymbol{\eta}_{o}\right)^{T} \dot{\boldsymbol{\eta}}_{o}
$$

where $E_{\eta}: S^{3} \rightarrow \mathbb{R}^{4 \times 3}$ is the matrix

$$
E_{\eta}(\boldsymbol{\eta}):=\left[\begin{array}{c}
-\boldsymbol{\epsilon}^{T} \\
\phi I_{3}-(\boldsymbol{\epsilon}) \times
\end{array}\right], \quad \forall \boldsymbol{\eta}=\left[\phi, \boldsymbol{\epsilon}^{T}\right]^{T} \in \mathrm{S}^{3} .
$$

The more practical consideration of rolling contacts, as opposed to a rigid grasp, requires no slip to occur between the agents and object by ensuring that each contact force remains inside the friction cone defined by:

$$
\mathscr{F}_{c_{i}}:=\left\{\boldsymbol{f}_{c_{i}}^{\mathcal{C}_{i}} \in \mathbb{R}^{3}: f_{n_{i}} \mu \geq \sqrt{f_{x_{i}}^{2}+f_{y_{i}}^{2}}\right\}
$$

where $\boldsymbol{f}_{c_{i}}^{\mathcal{C}_{i}}=\left(f_{x_{i}}, f_{y_{i}}, f_{n_{i}}\right)$ is the contact force at $i$ written in frame $\mathcal{C}_{i}$ with tangential force components $f_{x_{i}}, f_{y_{i}} \in \mathbb{R}$ and normal force component $f_{n_{i}} \in \mathbb{R}$, and $\mu \in \mathbb{R}_{>0}$ is the friction coefficient. The full friction cone is the Cartesian product of all the friction cones: $\mathscr{F}_{c}:=\mathscr{F}_{c_{1}} \times \ldots \times \mathscr{F}_{c_{n}}$.

When the contact points do not slip, the grasp relation $J_{h} \dot{\boldsymbol{q}}=\bar{G}^{T} \overline{\boldsymbol{v}}_{o}$ holds [8], which, after substituting [6], becomes:

$$
\boldsymbol{v}_{c}=J_{h} \dot{\boldsymbol{q}}=G^{T} \boldsymbol{v}_{o},
$$

where $\boldsymbol{v}_{c}:=\left[\boldsymbol{v}_{c_{1}}^{T}, \ldots, \boldsymbol{v}_{c_{N}}^{T}\right]^{T} \in \mathbb{R}^{3 n}$ is the vector of contact velocities.

The following assumptions are made for the grasp:

Assumption 1. The grasp consists of $N \geq 3$ agents with non-collinear contact points and $\mathfrak{N}(G) \cap \operatorname{Int}\left(\mathscr{F}_{c}\right) \neq \emptyset$.

Assumption 2. The matrix $J_{h}(\boldsymbol{q})$ is non-singular, and the contact points do not exceed the fingertip surface.

Remark 1. Note that $N \geq 3$ agents with non-collinear contact points ensures $G$ is full row rank [20]. The condition that $\mathfrak{N}(G) \bigcap \operatorname{Int}\left(\mathscr{F}_{c}\right) \neq \emptyset$ ensures the existence of a contact force that lies within the friction cone and yields a desired object wrench, which is called the force-closure condition [8]. Force-closure depends on the initial grasp, and can be ensured by existing high-level grasp planning methods [21]. Moreover, by incorporating optimization techniques, as e.g. in [22], we can enforce prevention of excessive rolling of the contacts and thus relax the respective part of Assumption 2. Finally, the non-singular condition of $J_{h}$ intuitively implies that tracking the desired reference trajectory does not force the agents through such singular configurations. This can also be achieved by exploiting internal motions of redundant agents $\left(n_{i}>3\right)$.

Moreover, the following Lemma will be needed in the subsequent analysis.

Lemma 1. [5] The matrices $M_{i}\left(\boldsymbol{q}_{i}\right), \bar{M}_{o}\left(\overline{\boldsymbol{\eta}}_{o}\right)$ are symmetric and positive-definite, and $\dot{M}_{i}\left(\boldsymbol{q}_{i}\right)-2 C_{i}\left(\boldsymbol{q}_{i}, \dot{\boldsymbol{q}}_{i}\right), \dot{\bar{M}}_{o}\left(\overline{\boldsymbol{\eta}}_{o}\right)-$ $2 \bar{C}_{o}\left(\overline{\boldsymbol{\eta}}_{o}, \overline{\boldsymbol{\omega}}_{o}\right)$ are skew-symmetric, $\forall \boldsymbol{q}_{i}, \dot{\boldsymbol{q}}_{i} \in \mathbb{R}^{n_{i}}, i \in$ $\mathscr{N}, \overline{\boldsymbol{\eta}}_{o}, \overline{\boldsymbol{\omega}}_{o} \in \mathbb{M} \times \mathbb{R}^{6}$.

In view of Lemma 1, one can verify that $M_{o}$ is positive definite and $\dot{M}_{o}-2 C_{o}$ is skew-symmetric as well. We also assume that the contact vectors $\boldsymbol{p}_{f c_{i}}^{\mathcal{F}_{i}}$ and $\dot{\boldsymbol{p}}_{f c_{i}}^{\mathcal{F}_{i}}$ are measured accurately online, $\forall i \in \mathscr{N}$. This can be achieved either by the use of appropriate tactile sensors or forward simulation of the contact dynamics.

Let now a desired pose trajectory, $\boldsymbol{p}_{\mathrm{d}}: \mathbb{R}_{\geq 0} \rightarrow \mathbb{R}^{3}, \boldsymbol{\eta}_{\mathrm{d}}:=$ $\left[\phi_{\mathrm{d}}, \epsilon_{\mathrm{d}}^{T}\right]^{T}: \mathbb{R}_{\geq 0} \rightarrow \mathrm{S}^{3}$, to be tracked by $\boldsymbol{x}_{o}$. To that end, we define the position error $\boldsymbol{e}_{p}:=\boldsymbol{p}_{o}-\boldsymbol{p}_{\mathrm{d}}$ as well as the quaternion product $\boldsymbol{e}_{\eta}:=\boldsymbol{\eta}_{\mathrm{d}} \otimes \boldsymbol{\eta}_{o}^{+}$, as an orientation error metric [5], where $\boldsymbol{\eta}^{+}:=\left[\phi,-\boldsymbol{\epsilon}^{T}\right]^{T}$ denotes the quaternion conjugate. The aim is then to regulate $\boldsymbol{e}_{\eta}$ to $\left[ \pm 1, \mathbf{0}_{3}^{T}\right]^{T}$ [5]. Moreover, we aim at ensuring that the fingers are always in contact with the object and slipping is avoided. Formally, the problem is defined as follows. 
Problem 1. Given a desired bounded, smooth object pose trajectory defined by $\boldsymbol{p}_{d}: \mathbb{R}_{\geq 0} \rightarrow \mathbb{R}^{3}, \boldsymbol{\eta}_{d}: \mathbb{R}_{\geq 0} \rightarrow \mathrm{S}^{3}$, with bounded first and second derivatives, determine a control law $\boldsymbol{u}$ in (2) such that the following conditions hold:

1) $\lim _{t \rightarrow \infty}\left(\boldsymbol{e}_{p}(t), \boldsymbol{e}_{\eta}(t)\right)=\left(\mathbf{0}_{3},\left[ \pm 1, \mathbf{0}_{3}^{T}\right]^{T}\right)$

2) $\boldsymbol{f}_{c_{i}}^{C_{i}}(t) \in \mathscr{F}_{c_{i}}, \forall t>0, i \in \mathscr{N}$.

\section{PROPOSED CONTROL SCHEME}

This section presents the proposed control scheme, which employs adaptive control techniques for the compensation of the uncertainties described in the previous section.

Without loss of generality, we assume that $n_{i}=3$, $\forall i \in \mathscr{N}$, i.e., the agents are not redundant. The proposed solution can be trivially extended to redundant cases, e.g., by following the analysis of [20, Chapter 6]. By combining the agent and object dynamics (2), (5) as well as (9), we can obtain the coupled dynamics

$$
\tilde{M} \dot{\boldsymbol{v}}_{o}+\tilde{C} \boldsymbol{v}_{o}+\tilde{\boldsymbol{g}}=G J_{h}^{-T} \boldsymbol{u},
$$

where $\tilde{M}:=\tilde{M}(\tilde{\boldsymbol{x}}):=M_{o}+G J_{h}^{-T} M J_{h}^{-1} G^{T}, \tilde{C}:=$ $\tilde{C}(\tilde{\boldsymbol{x}}, \dot{\tilde{\boldsymbol{x}}}):=C_{o}+G J_{h}^{-T}\left(C J_{h}^{-1} G^{T}+M \frac{d}{d t}\left(J_{h}^{-1} G^{T}\right)\right), \tilde{\boldsymbol{g}}:=$ $\tilde{\boldsymbol{g}}(\tilde{\boldsymbol{x}}):=\boldsymbol{g}_{o}+G J_{h}^{-T} \boldsymbol{g}$, and $\tilde{\boldsymbol{x}}:=\left[\boldsymbol{\eta}_{o}^{T}, \boldsymbol{q}^{T}, \boldsymbol{p}_{f c}^{T}, \boldsymbol{p}_{o c}^{T}\right]^{T} \in$ $\mathbb{T} \times \mathbb{R}^{n+6 N}$. The following lemma, which can be derived using Lemma 11, states useful properties of (10):

Lemma 2. [5] The matrix $\tilde{M}$, is symmetric and positivedefinite, and $\dot{\tilde{M}}-2 \tilde{C}$ is skew-symmetric.

Next, the left-hand side of the object dynamics is linearly parameterized with respect to the dynamic parameters as:

$$
M_{o}\left(\boldsymbol{\eta}_{o}\right) \dot{\boldsymbol{v}}_{o}+C_{o}\left(\boldsymbol{\eta}_{o}, \boldsymbol{\omega}_{o}\right) \boldsymbol{v}_{o}+\boldsymbol{g}_{o}=Y_{o}\left(\boldsymbol{\eta}_{o}, \boldsymbol{\omega}_{o}, \boldsymbol{v}_{o}, \dot{\boldsymbol{v}}_{o}\right) \boldsymbol{\nu}_{o}
$$

where $\boldsymbol{\nu}_{o} \in \mathbb{R}^{l_{o}}, l_{o} \in \mathbb{N}$, is a vector containing unknown object dynamic parameters as well as the term $\boldsymbol{p}_{\bar{O} O}^{\mathcal{O}}$, introduced in (4), and $Y_{o}: \mathbb{T} \times \mathbb{R}^{18} \rightarrow \mathbb{R}^{6 \times l_{o}}$ is a known regressor matrix.

Similarly, the part of (10) that concerns the robotic agents can be linearly parameterized as:

$$
\begin{gathered}
M J_{h}^{-1} G^{T} \dot{\boldsymbol{v}}_{o}+M\left(\frac{\partial}{\partial t}\left(J_{h}^{-1} G^{T}\right)+C J_{h}^{-1} G^{T}\right) \boldsymbol{v}_{o}+\boldsymbol{g}= \\
Y\left(\tilde{\boldsymbol{x}}, \dot{\tilde{\boldsymbol{x}}}, \boldsymbol{v}_{o}, \dot{\boldsymbol{v}}_{o}\right) \boldsymbol{\nu}
\end{gathered}
$$

where $\nu \in \mathbb{R}^{l}, l \in \mathbb{N}$, is a vector of unknown dynamic parameters of the agents, and $Y: \mathbb{T} \times \mathbb{R}^{2 n+15 N} \rightarrow \mathbb{R}^{6 N \times l}$ is the respective known regressor matrix.

Therefore, the left-hand side of the coupled dynamics (10) can be written as

$$
\begin{aligned}
& \tilde{M} \dot{\boldsymbol{v}}_{o}+\tilde{C} \boldsymbol{v}_{o}+\tilde{\boldsymbol{g}}= \\
& \quad Y_{o}\left(\boldsymbol{\eta}_{o}, \boldsymbol{\omega}_{o}, \boldsymbol{v}_{o}, \dot{\boldsymbol{v}}_{o}\right) \boldsymbol{\nu}_{o}+G J_{h}^{-T} Y\left(\tilde{\boldsymbol{x}}, \dot{\tilde{\boldsymbol{x}}}, \boldsymbol{v}_{o}, \dot{\boldsymbol{v}}_{o}\right) \boldsymbol{\nu}
\end{aligned}
$$

Let now $\hat{\boldsymbol{\nu}} \in \mathbb{R}^{l}, \hat{\boldsymbol{\nu}}_{o} \in \mathbb{R}^{l_{o}}$, be the estimates of $\boldsymbol{\nu}$ and $\boldsymbol{\nu}_{o}$, respectively, by the agents, and the respective errors $\boldsymbol{e}_{\nu}:=$ $\hat{\boldsymbol{\nu}}-\boldsymbol{\nu}$, and $\boldsymbol{e}_{\nu_{o}}:=\hat{\boldsymbol{\nu}}_{o}-\boldsymbol{\nu}_{o}$.
Regarding the pose errors, as described in Problem 1, these are $\boldsymbol{e}_{p}=\boldsymbol{p}_{o}-\boldsymbol{p}_{\mathrm{d}}$ and $\boldsymbol{e}_{\eta}=\boldsymbol{\eta}_{\mathrm{d}} \otimes \boldsymbol{\eta}_{o}^{+}$, which can be shown to satisfy [5]:

$$
\begin{aligned}
& \boldsymbol{e}_{\eta}=\left[\begin{array}{c}
e_{\phi} \\
\boldsymbol{e}_{\epsilon}
\end{array}\right]:=\left[\begin{array}{c}
\phi_{o} \phi_{\mathrm{d}}+\boldsymbol{\epsilon}_{o}^{T} \boldsymbol{\epsilon}_{\mathrm{d}} \\
\phi_{o} \boldsymbol{\epsilon}_{\mathrm{d}}-\phi_{\mathrm{d}} \boldsymbol{\epsilon}_{o}+\left(\boldsymbol{\epsilon}_{o}\right) \times \boldsymbol{\epsilon}_{\mathrm{d}}
\end{array}\right] \\
& \dot{\boldsymbol{e}}_{p}=\dot{\boldsymbol{p}}_{o}-\dot{\boldsymbol{p}}_{\mathrm{d}} \\
& \dot{\boldsymbol{e}}_{\eta}=\left[\begin{array}{c}
\dot{e}_{\phi} \\
\dot{\boldsymbol{e}}_{\epsilon}
\end{array}\right]=\left[\begin{array}{c}
\frac{1}{2} \boldsymbol{e}_{\epsilon}^{T} \boldsymbol{e}_{\omega} \\
-\frac{1}{2}\left(e_{\phi} I_{3}+\left(\boldsymbol{e}_{\epsilon}\right) \times\right) \boldsymbol{e}_{\omega}-\left(\boldsymbol{e}_{\epsilon}\right) \times \dot{\boldsymbol{\omega}}_{\mathrm{d}}
\end{array}\right],
\end{aligned}
$$

where $\boldsymbol{e}_{\omega}:=\boldsymbol{\omega}_{o}-\boldsymbol{\omega}_{\mathrm{d}} \in \mathbb{R}^{3}$ and $\boldsymbol{\omega}_{\mathrm{d}}=2 E\left(\boldsymbol{\eta}_{\mathrm{d}}\right) \dot{\boldsymbol{\eta}}_{\mathrm{d}}$, similarly to (7). Note that, except for $\left(\boldsymbol{e}_{p}, \boldsymbol{e}_{\eta}, \boldsymbol{e}_{\omega}\right)=$ $\left(\mathbf{0}_{3},\left[ \pm 1, \mathbf{0}_{3}^{T}\right]^{T}, \mathbf{0}_{3}\right)$ (the desired equilibirum), the point $\left(\boldsymbol{e}_{p}, \boldsymbol{e}_{\eta}, \boldsymbol{e}_{\omega}\right)=\left(\mathbf{0}_{3},\left[0, \tilde{\boldsymbol{e}}_{\epsilon}^{T}\right]^{T}, \mathbf{0}_{3}\right)$ is also an equilibrium of (12), for any $\tilde{\boldsymbol{e}}_{\epsilon} \in \mathrm{S}^{2}$. The latter represents an undesired local minimum of the dynamics (12) and stems from topological obstructions of the orientation space [23]. Hence, a continuous controller cannot achieve global stabilization of $\left(\boldsymbol{e}_{p}, \boldsymbol{e}_{\eta}, \boldsymbol{e}_{\omega}\right)$ to the desired equilibrium point. As will be shown later, however, we will achieve almost global stabilization, i.e., from all initial conditions other than the aforementioned undesired local minimum configurations that satisfy $e_{\phi}(0)=0$, which form a lower-dimensional manifold.

We provide next the proposed control protocol. First, we design the reference velocity signal $\boldsymbol{v}_{f} \in \mathbb{R}^{6}$ and the associated velocity error $\boldsymbol{e}_{v}$ as

$$
\begin{aligned}
& \boldsymbol{v}_{f}:=\boldsymbol{v}_{\mathrm{d}}-K \boldsymbol{e}:=\left[\begin{array}{c}
\dot{\boldsymbol{p}}_{\mathrm{d}} \\
\boldsymbol{\omega}_{\mathrm{d}}
\end{array}\right]-\left[\begin{array}{c}
k_{p} \boldsymbol{e}_{p} \\
-k_{\eta} \frac{\boldsymbol{e}_{\epsilon}}{e_{\phi}^{3}}
\end{array}\right] \\
& \boldsymbol{e}_{v}:=\boldsymbol{v}_{o}-\boldsymbol{v}_{f},
\end{aligned}
$$

where $K:=\operatorname{diag}\left\{k_{p} I_{3}, k_{\eta} I_{3}\right\} \in \mathbb{R}^{3}$ is a positive definite gain matrix, with $k_{p}, k_{\eta}$ positive constants, $\boldsymbol{e}:=\left[\boldsymbol{e}_{p}^{T},-\frac{\boldsymbol{e}_{\epsilon}^{T}}{e_{\phi}^{3}}\right]^{T}$, and $\boldsymbol{v}_{\mathrm{d}}:=\left[\dot{\boldsymbol{p}}_{\mathrm{d}}^{T}, \boldsymbol{\omega}_{\mathrm{d}}^{T}\right]^{T}$. As will be shown later, $e_{\phi}(0) \neq 0 \Rightarrow$ $e_{\phi}(t) \neq 0, \forall t \geq 0$, thus 13a is well defined.

We design now the control protocol as

$$
u=Y_{r} \hat{\boldsymbol{\nu}}+J_{h}^{T}\left(G^{*} \boldsymbol{f}_{\mathrm{d}}+\boldsymbol{f}_{\text {int }}\right),
$$

where $G^{*}$ is the Moore-Penrose pseudoinverse of $G, f_{\mathrm{d}}:=$ $Y_{o_{r}} \hat{\boldsymbol{\nu}}_{o}-\boldsymbol{e}-K_{v} \boldsymbol{e}_{v}$ with $K_{v} \in \mathbb{R}^{6}$ a positive definite gain matrix, $Y_{r}:=Y\left(\tilde{\boldsymbol{x}}, \dot{\tilde{\boldsymbol{x}}}, \boldsymbol{v}_{f}, \dot{\boldsymbol{v}}_{f}\right), Y_{o_{r}}:=Y_{o}\left(\boldsymbol{\eta}_{o}, \boldsymbol{\omega}_{o}, \boldsymbol{v}_{f}, \dot{\boldsymbol{v}}_{f}\right)$, and $f_{\text {int }}$ is a term in the nullspace of $G$ to prevent contact slip, which will be designed later. Moreover, we design the adaptation signals

$$
\begin{aligned}
\dot{\hat{\boldsymbol{\nu}}} & =\operatorname{Proj}\left(\hat{\boldsymbol{\nu}},-\Gamma Y_{r}^{T} J_{h}^{-1} G^{T} \boldsymbol{e}_{v}\right), \\
\dot{\hat{\boldsymbol{\nu}}}_{o} & =\operatorname{Proj}\left(\hat{\boldsymbol{\nu}}_{o},-\Gamma_{o} Y_{o_{r}}^{T} \boldsymbol{e}_{v}\right),
\end{aligned}
$$

where $\Gamma \in \mathbb{R}^{l \times l}, \Gamma_{o} \in \mathbb{R}^{l_{o} \times l_{o}}$ are positive definite constant gain matrices, and $\operatorname{Proj}()$ is the projection operator, which satisfies [24]:

$$
(\hat{\boldsymbol{\theta}}-\boldsymbol{\theta})^{T}\left(W^{-1} \operatorname{Proj}(\hat{\boldsymbol{\theta}}, W \boldsymbol{z})-\boldsymbol{z}\right) \leq \mathbf{0}_{l_{z}},
$$

for any symmetric positive definite $W \in \mathbb{R}^{l_{z} \times l_{z}}$, and $\forall \boldsymbol{\theta}, \hat{\boldsymbol{\theta}}, \boldsymbol{z} \in \mathbb{R}^{l_{z}}$, for some $l_{z} \in \mathbb{N}$. Moreover, by appropriately 
choosing the initial conditions of the estimates $\hat{\boldsymbol{\nu}}(0), \hat{\boldsymbol{\nu}}(0)$, we guarantee via the projection operator that $\hat{\boldsymbol{\nu}}(t), \hat{\boldsymbol{\nu}}_{o}(t)$ will stay uniformly bounded in predefined sets defined by finite constants $\overline{\hat{\boldsymbol{\nu}}}, \overline{\hat{\boldsymbol{\nu}}}_{o}$, i.e., $\|\hat{\boldsymbol{\nu}}(t)\| \leq \overline{\hat{\boldsymbol{\nu}}},\left\|\hat{\boldsymbol{\nu}}_{o}(t)\right\| \leq \overline{\hat{\boldsymbol{\nu}}}_{o}, \forall t \geq 0$. Hence, we can achieve the boundedness of the respective errors as

$$
\begin{aligned}
\left\|\boldsymbol{e}_{\nu}(t)\right\| \leq \bar{e}_{\nu} & :=\overline{\hat{\boldsymbol{\nu}}}+\|\boldsymbol{\nu}\| \\
\left\|\boldsymbol{e}_{\nu_{o}}(t)\right\| \leq \bar{e}_{\nu_{o}} & :=\overline{\hat{\boldsymbol{\nu}}}_{o}+\left\|\boldsymbol{\nu}_{o}\right\|,
\end{aligned}
$$

for finite constants $\bar{e}_{\nu}, \bar{e}_{\nu_{o}}$. More details can be found in [24, Chapter 11].

We design next the internal force component $f_{\text {int }}$ to guarantee slip prevention. Slip is addressed by ensuring the contact forces remain inside the friction cone as specified in (8). The friction cone can be approximated as a pyramid with $n_{s} \in \mathbb{N}_{>3}$ sides, which results in a linear constraint with respect to the contact forces [18], [25]: $\Lambda_{i}(\mu) R_{p c_{i}}^{T} \boldsymbol{f}_{c_{i}} \succeq$ $0, \forall i \in \mathscr{N}$, or in vector form,

$$
\Lambda(\mu) R_{p c}^{T} \boldsymbol{f}_{c} \succeq 0
$$

where $R_{p c}:=\operatorname{diag}\left\{\left[R_{p c_{i}}\right]_{i \in \mathscr{N}}\right\}$, with $R_{p c_{i}} \in S O(3)$ the rotation matrices mapping the contact frames $\mathcal{C}_{i}$ to the inertial frame $\mathcal{P}, \Lambda(\mu):=\operatorname{diag}\left\{\left[\Lambda_{i}(\mu)\right]_{i \in \mathscr{N}}\right\}$, with $\Lambda_{i}(\mu) \in$ $\mathbb{R}^{n_{s} \times 3}$ define the pyramid matrices, and $\mu \in \mathbb{R}_{>0}$ is the friction coefficient.

The design of the internal force component, $f_{\text {int }}$, to ensure (18) is performed as follows. First, $f_{\text {int }}$ must be in the nullspace of $G$, i.e., $G \boldsymbol{f}_{\text {int }}=0$. Second, the internal force must satisfy (18) such that $\Lambda(\mu) R_{p c}^{T} f_{\text {int }} \succeq 0$. Third, the normal component of the internal force with respect to the contact plane must always be positive (i.e. the manipulators cannot "pull" on the contact point). To enforce this condition we design ${ }^{1} f_{\text {int }_{i}}=f_{\text {int }}^{\prime} R_{p c_{i}} \ell_{i}$, where $\ell_{i}:=\left[\ell_{i_{x}}, \ell_{i_{y}}, \ell_{i_{z}}\right]^{T}$ is the internal force direction in the contact frame $\mathcal{C}_{i}, i \in \mathscr{N}$, and $f_{\text {int }}^{\prime} \in \mathbb{R}_{>0}$ is a gain parameter to be designed. Without loss of generality let $\ell_{i_{z}}$ be aligned with the normal direction of the contact frame such that $\ell_{i_{z}}>0, i \in \mathscr{N}$, ensures that only pushing forces are applied at each contact. Satisfaction of the aforementioned conditions is done by solving the following convex quadratic program to define the internal force controller

$$
\begin{gathered}
\boldsymbol{f}_{\text {int }}=f_{\text {int }}^{\prime} R_{p c} \ell^{*} \\
\ell^{*}=\operatorname{argmin}_{\ell}\left\{\sum_{i \in \mathscr{N}} \ell_{i_{x}}^{2}+\ell_{i_{y}}^{2}+\ell_{i_{z}}^{2}\right\} \\
\text { s. t. } \\
G R_{p c} \ell=\mathbf{0}_{6}, \\
\ell_{i_{z}}>0, \quad \forall i \in \mathscr{N}, \\
\Lambda_{i}(\mu) \ell_{i} \succ \mathbf{0}_{n_{s}}, \forall i \in \mathscr{N},
\end{gathered}
$$

where $\ell:=\left[\ell_{1}^{T}, \ldots, \ell_{N}^{T}\right]^{T}$. Note that, since the contact points form a force-closure configuration, (19) always has a feasible solution.

\footnotetext{
${ }^{1}$ We use the notation $\boldsymbol{f}_{\text {int }}=\left[\boldsymbol{f}_{\text {int }_{1}}^{T}, \ldots, \boldsymbol{f}_{\text {int }_{N}}^{T}\right]^{T}$.
}

Finally, to satisfy $18, f_{\text {int }}$ must apply sufficient force inside the friction cone to reject perturbations that will arise during the manipulation motion that can push the contact force outside of the friction cone. Rejection of these perturbations is performed by designing the gain $f_{\text {int }}^{\prime}$ as follows. For simplicity we define the terms $\boldsymbol{k}=\Lambda(\mu) R_{p c}^{T} G^{*} \boldsymbol{f}_{\mathrm{d}}, \boldsymbol{l}=$ $\Lambda(\mu) \boldsymbol{\ell}$, and we denote by $k_{j}$ and $l_{j}$ the $j$ th element of $\boldsymbol{k}$ and $\boldsymbol{l}$ respectively for $j \in\left\{1, \ldots, N n_{s}\right\}$.

Noting that $\Lambda(\mu) \ell \succ 0$ from (19), we define the decreasing function $\kappa: \mathbb{R} \rightarrow \mathbb{R}_{\geq 0}$ as

$$
\kappa(x):= \begin{cases}-x, & \text { if } x \leq-1 \\ q(x), & \text { if }-1 \leq x \leq 0 \\ 0, & \text { if } x \geq 0\end{cases}
$$

where $q(x) \geq 0, \forall x \in[-1,0]$, is an appropriate polynomial that ensures continuous differentiability of $\kappa$, for instance $q(x)=x^{3}+2 x^{2}$. Then one can verify that $\kappa(x)+1 \geq$ $-x, \forall x \in \mathbb{R}$. We now design the magnitude scaling for the internal forces as

$$
f_{\text {int }}^{\prime}=\frac{\kappa\left(\min _{j}\left\{k_{j}\right\}\right)+1+\varepsilon}{\min _{j}\left\{l_{j}\right\}},
$$

where $\varepsilon \in \mathbb{R}_{>0}$ is a tuning gain. The intuition behind (20) is to upper bound elements of the control and the system dynamics to prevent either from pushing the contact force outside of the friction cone. The term $\kappa\left(\min _{j}\left\{k_{j}\right\}\right)+1$ cancels out any effects from $\boldsymbol{f}_{d}$. The term $\varepsilon$ handles the system dynamics, which is guaranteed to be bounded in the following theorem.

Remark 2. The internal force control presented here accounts for the dynamics of the system by appropriately scaling $f_{\text {int }}^{\prime}$, which rejects perturbations from causing slip. However, as opposed to [18], we relax the condition that $\varepsilon$ must upper bound all of the dynamics terms by exploiting knowledge of the applied controller via the term $\kappa\left(\min _{j}\left\{k_{j}\right\}\right)$. This reduces the amount of squeezing force applied to prevent crushing the object.

The stability and slip prevention guarantees of the proposed controller are presented in the following theorem.

Theorem 1. Consider $N$ robotic agents in contact with an object, described by the dynamics (2), (5), and suppose Assumptions 1 and 2 hold. Let the desired object pose $\left(\boldsymbol{p}_{\mathrm{d}}\right.$, $\left.\boldsymbol{\eta}_{\mathrm{d}}\right): \mathbb{R}_{\geq 0} \rightarrow \mathbb{R}^{3} \times \mathrm{S}^{3}$ be bounded with bounded first and second derivatives. Moreover, assume that $e_{\phi}(0) \neq 0$ and $\boldsymbol{f}_{c_{i}}^{C_{i}}(0) \in \mathscr{F}_{c_{i}}, \forall i \in \mathscr{N}$. Then, the control protocol (13a)-20) guarantees that $\lim _{t \rightarrow \infty}\left(\boldsymbol{e}_{p}(t), \boldsymbol{e}_{\eta}(t)\right)=$ $\left(\mathbf{0}_{3},\left[ \pm 1, \mathbf{0}_{3}^{T}\right]^{T}\right)$, as well as boundedness of all closed-loop signals. Moreover, by choosing a sufficiently large $\varepsilon$ in 20, it holds that $\boldsymbol{f}_{c}^{C_{i}}(t) \in \mathscr{F}_{c_{i}}, \forall t>0, i \in \mathscr{N}$.

Proof. Consider the stack vector state $\chi:=$ $\left[\boldsymbol{e}_{p}^{T}, \boldsymbol{e}_{\epsilon}^{T}, \boldsymbol{e}_{v}^{T}, \boldsymbol{e}_{\nu}^{T}, \boldsymbol{e}_{\nu_{o}}^{T}\right]^{T} \in \mathscr{X}:=\mathbb{R}^{12+\ell+\ell_{o}}$. Next, note by (2), (3), and (9) that, when $f_{c_{i}}^{C_{i}}(\chi) \in \mathscr{F}_{c_{i}}$, each $f_{c_{i}}^{C_{i}}$ can be written as a function of the stack state, i.e., 
$f_{c_{i}}^{C_{i}}=\boldsymbol{f}_{c_{i}}^{C_{i}}(\chi), \forall i \in \mathscr{N}$. Consider also the set

$$
\begin{aligned}
\mathscr{U}:= & \left\{\boldsymbol{\chi} \in \mathscr{X}:\left\|\boldsymbol{e}_{\epsilon}\right\|<\bar{e}_{\epsilon},\left\|\boldsymbol{e}_{p}\right\|<\bar{e}_{p},\left\|\boldsymbol{e}_{v}\right\|<\bar{e}_{v},\right. \\
& \left.\left\|\boldsymbol{e}_{\nu}\right\|<\tilde{e}_{\nu},\left\|\boldsymbol{e}_{\nu_{o}}\right\|<\tilde{e}_{\nu_{o}}, \boldsymbol{f}_{c_{i}}^{C_{i}}(\boldsymbol{\chi}) \in \operatorname{Int}\left(\mathscr{F}_{c_{i}}\right), \forall i \in \mathscr{N}\right\},
\end{aligned}
$$

for some positive constants $\bar{e}_{\epsilon}>1, \bar{e}_{v}, \bar{e}_{p}$ satisfying $\left\|\boldsymbol{e}_{v}(0)\right\|<\bar{e}_{v},\left\|\boldsymbol{e}_{p}(0)\right\|<\bar{e}_{p}$, and $\tilde{e}_{\nu}, \tilde{e}_{\nu_{o}}$ larger than $\bar{e}_{\nu}, \bar{e}_{\nu_{o}}$, respectively, which were introduced in (17). Note that $\chi(0) \in$ $\mathscr{U}$. Next, by using (14) and (15), one obtains the closed-loop dynamics $\dot{\chi}=\boldsymbol{h}_{\chi}(\boldsymbol{\chi}, t)$, where $\boldsymbol{h}_{\chi}: \mathscr{X} \times \mathbb{R}_{\geq 0} \rightarrow \mathscr{X}$ is a function that is continuous in $t$ and locally Lipschitz in $\chi$. Then, according to Theorem 2.1.3 of [26], there exists a positive time constant $\tau>0$ and a unique solution $\chi$ : $[0, \tau) \rightarrow \mathscr{U}$, i.e., defined for $[0, \tau)$ and satisfying $\chi(t) \in \mathscr{U}$, $\forall t \in[0, \tau)$. Hence, slip is prevented and the dynamics (10] are well-defined, for $t \in[0, \tau)$.

Let now the Lyapunov function

$$
V:=\frac{1}{2} \boldsymbol{e}_{p}^{T} \boldsymbol{e}_{p}+\frac{2}{e_{\phi}^{2}}+\frac{1}{2} \boldsymbol{e}_{v}^{T} \tilde{M} \boldsymbol{e}_{v}+\frac{1}{2} \boldsymbol{e}_{\nu}^{T} \Gamma^{-1} \boldsymbol{e}_{\nu}+\frac{1}{2} \boldsymbol{e}_{\nu_{o}}^{T} \Gamma_{o}^{-1} \boldsymbol{e}_{\nu_{o}} .
$$

Since $e_{\phi}(0) \neq 0$, it holds that $V(0) \leq \bar{V}_{0}$ for a finite positive $\bar{V}_{0}$. Differentiation of $V$ results in:

$$
\begin{array}{r}
\dot{V}=\boldsymbol{e}^{T}\left(\boldsymbol{v}_{o}-\boldsymbol{v}_{\mathrm{d}}\right)+\frac{1}{2} \boldsymbol{e}_{v}^{T} \dot{\tilde{M}} \boldsymbol{e}_{v}+\boldsymbol{e}_{v}^{T}\left(-\tilde{C} \boldsymbol{v}_{o}-\tilde{\boldsymbol{g}}-\tilde{M} \dot{\boldsymbol{v}}_{f}\right. \\
\left.+G J_{h}^{-T} \boldsymbol{u}\right)+\boldsymbol{e}_{\nu}^{T} \Gamma^{-1} \dot{\boldsymbol{e}}_{\nu}+\boldsymbol{e}_{\nu_{o}}^{T} \Gamma_{o}^{-1} \dot{\boldsymbol{e}}_{\nu_{o}}
\end{array}
$$

Exploitation of the skew symmetry of $\dot{\tilde{M}}-2 \tilde{C}$ system, use of $\boldsymbol{v}_{o}=\boldsymbol{e}_{v}+\boldsymbol{v}_{f}$, use of [11), and substitution of the control law (14) results in:

$$
\begin{array}{r}
\dot{V}=-\boldsymbol{e}^{T} K \boldsymbol{e}-\boldsymbol{e}_{v}^{T} K_{v} \boldsymbol{e}_{v}+\boldsymbol{e}_{v}^{T}\left(Y_{o_{r}} \boldsymbol{e}_{\nu_{o}}+G J_{h}^{-T} Y_{r} \boldsymbol{e}_{\nu}\right) \\
+\boldsymbol{e}_{\nu}^{T} \Gamma^{-1} \dot{\boldsymbol{e}}_{\nu}+\boldsymbol{e}_{\nu_{o}}^{T} \Gamma_{o}^{-1} \dot{\boldsymbol{e}}_{\nu_{o}}
\end{array}
$$

where we used the fact that $G \boldsymbol{f}_{\text {int }}=\mathbf{0}_{6}$ through (19). Finally, by substituting the adaptation laws 15 , we obtain

$$
\begin{gathered}
\dot{V}=-\boldsymbol{e}^{T} K \boldsymbol{e}-\boldsymbol{e}_{v}^{T} K_{v} \boldsymbol{e}_{v}+\boldsymbol{e}_{\nu}^{T}\left(\Gamma^{-1} \operatorname{Proj}\left(\hat{\boldsymbol{\nu}},-Y_{r}^{T} J_{h}^{-1} G^{T} \boldsymbol{e}_{v}\right)\right. \\
\left.+\Gamma Y_{r}^{T} J_{h}^{-1} G^{T} \boldsymbol{e}_{v}\right)+\boldsymbol{e}_{\nu_{o}}^{T}\left(\Gamma_{o}^{-1} \operatorname{Proj}\left(\hat{\boldsymbol{\nu}}_{o},-Y_{o_{r}}{ }^{T} \boldsymbol{e}_{v}\right)+\Gamma_{o} Y_{o_{r}}^{T} \boldsymbol{e}_{v}\right)
\end{gathered}
$$

which, by invoking the projection operator property (16) becomes $\dot{V} \leq-\boldsymbol{e}^{T} K \boldsymbol{e}-\boldsymbol{e}_{v}^{T} K_{v} \boldsymbol{e}_{v}$. Thus $\dot{V}$ is negative semidefinite, and $V$ is bounded in a compact set as $V(t) \leq V(0)$, $\forall t \in[0, \tau)$. In addition, $e_{\phi}(t) \neq 0, \forall t \in[0, \tau)$. Hence, the terms $\boldsymbol{e}_{p}(t), \boldsymbol{e}_{\epsilon}(t), e_{\phi}(t)$ are bounded in a compact set defined by $V(0)$ and not dependent on $\tau, \forall t \in[0, \tau)$. Therefore, since $\boldsymbol{p}_{\mathrm{d}}(t)$ and $\boldsymbol{\eta}_{\mathrm{d}}(t)$ are bounded and have bounded derivatives, one concludes that $\boldsymbol{p}_{o}(t), \boldsymbol{\eta}_{o}(t) \boldsymbol{v}_{o}(t)$, and $\boldsymbol{v}_{f}(t), \dot{\boldsymbol{v}}_{f}(t)$ are also bounded in compact sets, $\forall t \in$ $[0, \tau)$. This also implies boundedness of $\tilde{\boldsymbol{x}}, \dot{\tilde{\boldsymbol{x}}}$, as introduced in (10), which, along with Assumption 2 and properties of Euler-Lagrange systems [27], implies that $Y\left(\tilde{\boldsymbol{x}}, \dot{\tilde{\boldsymbol{x}}}, \boldsymbol{v}_{o}, \dot{\boldsymbol{v}}_{o}\right)$, $Y_{r}, Y_{o}\left(\boldsymbol{\eta}_{o}, \boldsymbol{\omega}_{o}, \boldsymbol{e}_{v}, \dot{\boldsymbol{e}}_{v}\right), Y_{o_{r}}$ are also bounded in compact sets that are independent of $\tau, \forall t \in[0, \tau)$. We prove next the slip prevention using the design of the internal force component

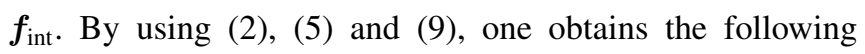
expression for the interaction forces:

$$
\begin{array}{r}
\boldsymbol{f}_{c}=B^{-1}\left(J _ { h } M ^ { - 1 } \left[\boldsymbol{u}-\boldsymbol{g}-\left(C J_{h}^{-1} G^{T}+\right.\right.\right. \\
\left.\left.\left.M \frac{d}{d t}\left(J_{h}^{-1} G^{T}\right)\right) \boldsymbol{v}_{o}\right]+G^{T} M_{o}^{-1}\left(C_{o} \boldsymbol{v}_{o}+\boldsymbol{g}_{o}\right)\right)
\end{array}
$$

where $B:=J_{h} M^{-1} J_{h}^{T}+G^{T} M_{o}^{-1} G$, which, by replacing $\boldsymbol{u}$, using $\boldsymbol{v}_{f}=\boldsymbol{e}_{v}+\boldsymbol{v}_{o}$ and (11), adding and subtracting $B^{-1} G^{T} M_{o}^{-1} \boldsymbol{f}_{\mathrm{d}}=B^{-1} G^{T} M_{o}^{-1} G G^{*} \boldsymbol{f}_{o, d}$ and adding $B^{-1} G^{T} M_{o}^{-1} G \boldsymbol{f}_{\text {int }}=\mathbf{0}_{3 N}$, becomes

$$
\boldsymbol{f}_{c}=G^{*} \boldsymbol{f}_{\mathrm{d}}+\boldsymbol{f}_{\mathrm{int}}+\boldsymbol{h}
$$

where $\boldsymbol{h}:=B^{-1} J_{h} M^{-1}\left(Y\left(\tilde{\boldsymbol{x}}, \dot{\tilde{\boldsymbol{x}}}, \boldsymbol{e}_{v}, \dot{\boldsymbol{e}}_{v}\right) \nu+Y_{r} \boldsymbol{e}_{\nu}\right)+$ $B^{-1} G^{T} M_{o}\left(\boldsymbol{e}+K_{v} \boldsymbol{e}_{v}-Y_{o}\left(\boldsymbol{e}_{o}, \boldsymbol{\omega}_{o}, \boldsymbol{e}_{v}, \dot{\boldsymbol{e}}_{v}\right) \nu_{o}-Y_{o_{r}} \boldsymbol{e}_{\nu_{o}}\right)$.

By combining the aforementioned expression with (18), one obtains the following condition for slip prevention:

$$
\Lambda(\mu) R_{p c}^{T} f_{\text {int }} \succeq-\Lambda(\mu) R_{p c}^{T} G^{*} f_{\mathrm{d}}-\Lambda(\mu) R_{p c}^{T} \boldsymbol{h} .
$$

Note that due to the aforementioned Lyapunov analysis, as well as the adaptation laws (15) through the projection operator, $\boldsymbol{e}, \boldsymbol{e}_{v}(t), \dot{\boldsymbol{e}}_{v}(t), \boldsymbol{e}_{\nu}(t), \boldsymbol{e}_{\nu_{o}}(t)$ are bounded in compact set independent of $\tau, \forall t \in[0, \tau)$. By combining this with the aforementioned analysis, we conclude that that $\boldsymbol{h}$ is bounded for all $\forall t \in[0, \tau)$ in a compact set, independent of $\tau$. Hence, by denoting $\varepsilon_{h}$ the maximum bound of the elements of $\pm \Lambda(\mu) R_{p c}^{T} \boldsymbol{h}$ and using the designed internal force component $f_{\text {int }}=f_{\text {int }}^{\prime} R_{p c} \ell$, a sufficient condition for (22) to hold is for the $j$ th element to satisfy

$$
l_{j} f_{\text {int }}^{\prime} \geq-k_{j}+\varepsilon_{h},
$$

$\forall j \in\left\{1, \ldots, N n_{s}\right\}$. By substituting 20, the left side satisfies

$l_{j} \frac{\kappa\left(\min _{j}\left\{k_{j}\right\}\right)+1+\varepsilon}{\min _{j}\left\{l_{j}\right\}} \geq \kappa\left(\min _{j}\left\{k_{j}\right\}\right)+1+\varepsilon \geq-k_{j}+\varepsilon$,

where we use $\kappa(x) \geq 0, \kappa(x)+1 \geq-x, \forall x \in \mathbb{R}$, and $\kappa\left(\min _{j}\left(k_{j}\right)\right)>\kappa\left(k_{j}\right), \forall j \in\left\{1, \ldots, N n_{s}\right\}$, since $\kappa()$ is decreasing. Hence, by choosing a large enough $\varepsilon$ we guarantee $\varepsilon \geq \varepsilon_{h}$ and hence contact slip is avoided $\forall t \in[0, \tau)$. In fact, the internal forces analysis above and the fact that $\Lambda$ defines pyramid constraints imply that $\boldsymbol{f}_{c_{i}}^{\mathcal{C}_{i}} \in \overline{\mathscr{F}}_{c_{i}}$, where $\overline{\mathscr{F}}_{c_{i}}$ is a compact subset of $\operatorname{Int}\left(\mathscr{F}_{c_{i}}\right), \forall i \in \mathscr{N}$. Therefore, since $\boldsymbol{e}_{\nu}$ and $\boldsymbol{e}_{\nu_{o}}$ are uniformly bounded through the projection operator by $\bar{e}_{\nu}$ and $\bar{e}_{\nu_{o}}$, respectively, by choosing large enough $\bar{e}_{p}$ and $\bar{e}_{v}$ in the definition of $\mathscr{U}, \chi(t)$ belongs to a compact subset $\overline{\mathscr{U}}$ of $\mathscr{U}, \forall t \in[0, \tau)$. Thus by invoking the maximal solutions' theorem (e.g., Th. 2.1.4 of [26]), it follows that $\tau=\infty$.

Note, finally, that $\boldsymbol{u}(t)$, as designed in (14), is bounded, $\forall t \geq 0$. Therefore, one can conclude that $\dot{e}_{v}(t)$ and thus $\ddot{\boldsymbol{q}}(t)$ is bounded, $\forall t \geq 0$. Hence, it follows that $\ddot{V}(t)$ is also bounded, $\forall t \geq 0$. Thus by invoking Barbalat's lemma (Lemma 8.2 of [28]), it follows that $\lim _{t \rightarrow \infty} \dot{V}(t)=0$ and so $\lim _{t \rightarrow \infty} \boldsymbol{e}(t) \rightarrow \mathbf{0}_{6}$ and $\lim _{t \rightarrow \infty} \boldsymbol{e}_{v}(t) \rightarrow \mathbf{0}_{6}$. This implies that $\lim _{t \rightarrow \infty} \boldsymbol{e}_{\epsilon}(t) \rightarrow \mathbf{0}_{3}$, which, given that $\boldsymbol{e}_{\eta}$ is 
a unit quaternion and $e_{\phi}(t) \neq 0, \forall t \geq 0$, ensures asymptotic stability of the pose error as $\lim _{t \rightarrow \infty}\left(\boldsymbol{e}_{p}(t), \boldsymbol{e}_{\eta}(t)\right)=$ $\left(\mathbf{0}_{3},\left[\operatorname{sgn}\left(e_{\phi}(0)\right), \mathbf{0}_{3}^{T}\right]^{T}\right)$.

Remark 3. Note that the bound $\varepsilon_{h}$ of $\boldsymbol{h}$ in (21) can be computed a priori. In practice, the terms $\nu, \nu_{o}$, which concern masses and moments of inertia of the object and the agents, can be known a priori up to a certain accuracy, leading thus to respective bounds. Hence, one can compute upper bounds for $V(0)$ and hence for $\boldsymbol{e}, \boldsymbol{e}_{v}, \boldsymbol{e}_{\nu}$, and $\boldsymbol{e}_{\nu_{o}}$. Since the structure of the dynamic terms is known, this can also lead to a bound of the terms $B^{-1}, M^{-1}, M_{o}, Y(\cdot)$, $Y_{o}(\cdot), Y_{r}$, and $Y_{o_{r}}$ that appear in $\boldsymbol{h}$. Hence, tuning of $\varepsilon$ to overcome $\varepsilon_{h}$ can be performed off-line.

\section{SIMULATION RESULTS}

The proposed control algorithm ensures asymptotic stability for cooperative manipulation with rolling contacts, as well as no slip, while being robust to dynamic uncertainties of the object-robot system. In this section, we implement the proposed control scheme on three $6 \mathrm{DOF}$ mobile manipulators consisting of a $3 \mathrm{DOF}, 3 \mathrm{~kg}$ base (X-Y translation, rotation about $\mathrm{Z}$ ) and a $3 \mathrm{DOF}$ manipulator with 3 identical links of length $0.3 \mathrm{~m}$ and mass of $0.5 \mathrm{~kg}$ each, as depicted in Fig. 2. The objective is to transport a $2 \mathrm{~kg}$ box along the desired reference trajectory defined by $\boldsymbol{p}_{\mathrm{d}}(t):=$ $[0.1 \sin (0.125 t), 0.1 \sin (0.125 t), 0.1 \sin (0.125 t)]^{T}, \boldsymbol{\eta}_{\mathrm{d}}(t):=$ $[\cos (0.1 \sin (.125 t)), 0,0, \sin (0.1 \sin (0.125 t))]^{T}$. The control gains used are: $k_{p}=1, k_{\eta}=.5, K_{v}=\operatorname{diag}[5,5,5,2,2,2]$, $\varepsilon=0.1, \Gamma_{o}=0.5 I_{l o \times l o}, \Gamma=0.5 I_{l \times l}$. The control is implemented with $30 \%$ error in all uncertain parameter (including the object center of mass), and the projection operator enforces the following bounds on the uncertain terms: $\overline{\hat{\nu}}=2.25, \overline{\hat{\nu}}_{o}=1.5$.

The simulation results are depicted in Figs. 3 7 for 50 seconds. More specifically, Figs. 3 and 4 show the resulting error trajectories of the object-agent system, which satisfy $\lim _{t \rightarrow \infty} \boldsymbol{e}_{p}(t)=\mathbf{0}_{3}, \lim _{t \rightarrow \infty} \boldsymbol{e}_{\varepsilon}(t)=\mathbf{0}_{3}$, and $\lim _{t \rightarrow \infty} \boldsymbol{e}_{\phi}(t)=\operatorname{sgn}\left(\boldsymbol{e}_{\phi}(0)\right)=1$ in the presence of rolling effects. Fig. 5 illustrates the boundedness of the uncertain parameters, $\hat{\nu}_{\boldsymbol{\nu}} \hat{\boldsymbol{\nu}}_{o}$ that is enforced by the proposed control scheme. Fig. 6 shows the required friction, $\mu_{r i}:=\frac{\sqrt{f_{x_{i}}^{2}+f_{y_{i}}^{2}}}{f_{n_{i}}}$, which denotes the minimum friction coefficient necessary to prevent slip throughout the motion [18]. If the required friction surpasses the true coefficient, then the contact point will slip and the grasp is compromised. As shown in Fig. 6. however, the required friction for each contact is below the true coefficient of $\mu=0.9$, which indicates that slip is prevented as guaranteed by the proposed method. Finally, Fig. 7 depicts the control inputs of the agents. As predicted by the theoretical analysis, asymptotic error stability as well as contact slip prevention are achieved.

\section{CONCLUSION AND FUTURE WORK}

This paper presented a control scheme for robust cooperative manipulation with rolling contacts. No information

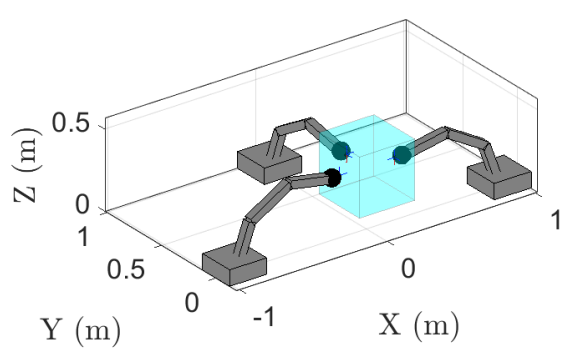

Fig. 2: Initial configuration of the system that consists of three mobile manipulators and a rigid object.

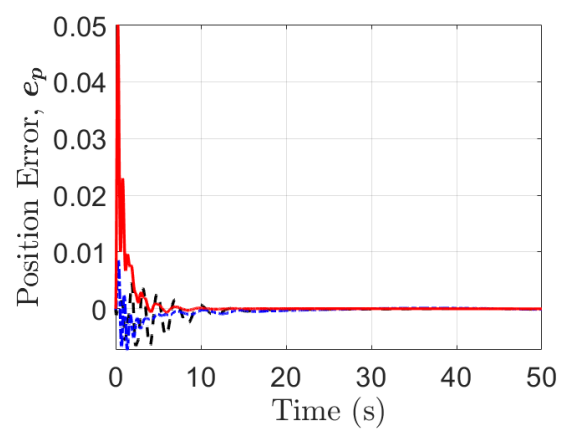

Fig. 3: The evolution of the position error, $\boldsymbol{e}_{p}(t), \forall t \in[0,50]$.

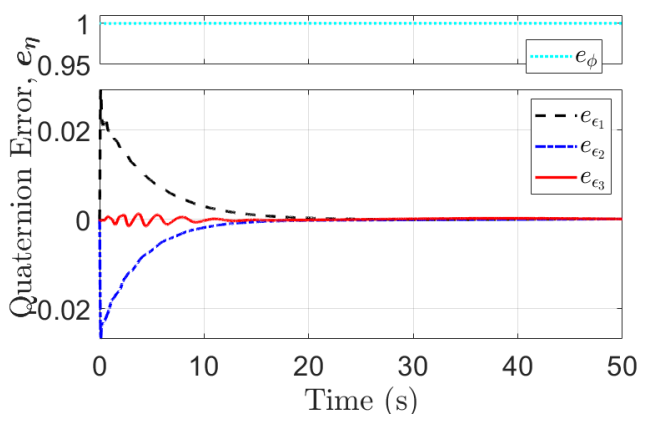

Fig. 4: The evolution of $e_{\phi}(t), e_{\epsilon}(t), \forall t \in[0,50]$.
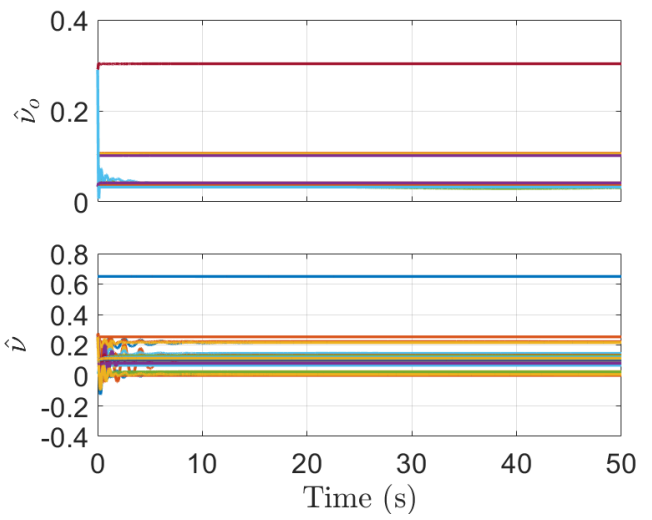

Fig. 5: The evolution of $\hat{\boldsymbol{\nu}}_{o}(t), \hat{\boldsymbol{\nu}}(t), \forall t \in[0,50]$. 


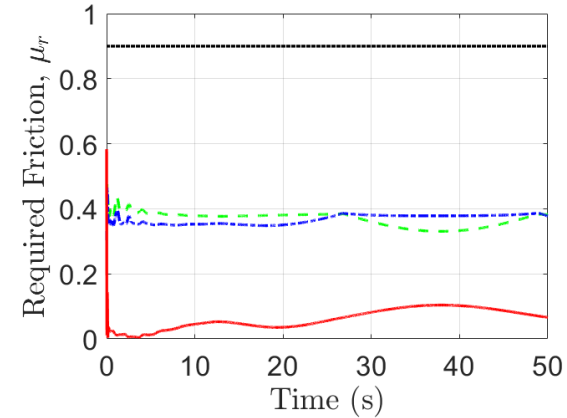

Fig. 6: The required friction to prevent slip for the three agents. The black dashed line represents $\mu=0.9$.

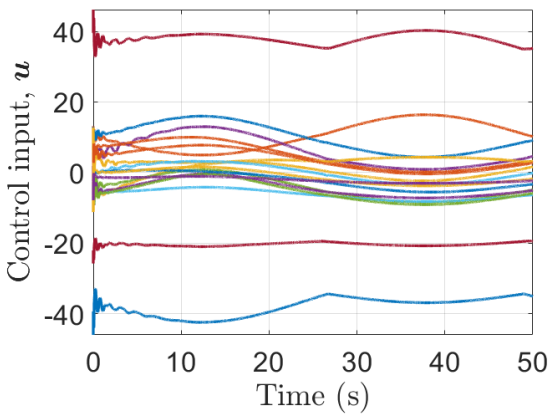

Fig. 7: The resulting inputs $\boldsymbol{u}(t)$ of the agents, $\forall t \in[0,50]$.

regarding the interaction object/agents forces was used, and an adaptive control methodology was employed to compensate for the unknown dynamic parameters as well as the location of the object center of mass. Finally, a novel internal force scheme was introduced for contact slip prevention. Future directions will address grasp reconfiguration as well as decentralization of the control protocol.

\section{REFERENCES}

[1] S. Erhart, D. Sieber, and S. Hirche, "An impedance-based control architecture for multi-robot cooperative dual-arm mobile manipulation," IEEE/RSJ International Conference on Intelligent Robots and Systems (IROS), pp. 315-322, 2013.

[2] F. Ficuciello, A. Romano, L. Villani, and B. Siciliano, "Cartesian impedance control of redundant manipulators for human-robot co-manipulation," IEEE/RSJ International Conference on Intelligent Robots and Systems (IROS), pp. 2120-2125, 2014.

[3] A.-N. Ponce-Hinestroza, J.-A. Castro-Castro, H.-I. Guerrero-Reyes, V. Parra-Vega, and E. Olguín-Díaz, "Cooperative redundant omnidirectional mobile manipulators: Model-free decentralized integral sliding modes and passive velocity fields," IEEE International Conference on Robotics and Automation (ICRA), pp. 2375-2380, 2016.

[4] Y.-H. Liu and S. Arimoto, "Decentralized adaptive and nonadaptive position/force controllers for redundant manipulators in cooperations," The International Journal of Robotics Research, vol. 17, no. 3, pp. 232-247, 1998.

[5] C. K. Verginis, M. Mastellaro, and D. V. Dimarogonas, "Robust cooperative manipulation without force/torque measurements: Control design and experiments," IEEE Transactions on Control Systems Technology, 2019.

[6] R. Ozawa and K. Tahara, "Grasp and dexterous manipulation of multifingered robotic hands: a review from a control view point," Advanced Robotics, vol. 31, no. 19-20, pp. 1030-1050, 2017.

[7] J. Kerr and B. Roth, "Analysis of multifingered hands," The International Journal of Robotics Research, vol. 4, no. 4, pp. 3-17, 1986.
[8] A. B. Cole, J. E. Hauser, and S. S. Sastry, "Kinematics and control of multifingered hands with rolling contact," IEEE Transactions on Automatic Control, vol. 34, no. 4, pp. 398-404, 1989.

[9] Y. Fan, L. Sun, M. Zheng, W. Gao, and M. Tomizuka, "Robust dexterous manipulation under object dynamics uncertainties," IEEE International Conference on Advanced Intelligent Mechatronics (AIM), pp. 613-619, 2017.

[10] A. Caldas, A. Micaelli, M. Grossard, M. Makarov, P. RodriguezAyerbe, and D. Dumur, "Object-level impedance control for dexterous manipulation with contact uncertainties using an LMI-based approach," IEEE International Conference on Robotics and Automation (ICRA), pp. 3668-3674, 2015.

[11] T. Wimböck, C. Ott, A. Albu-Schäffer, and G. Hirzinger, "Comparison of object-level grasp controllers for dynamic dexterous manipulation," The International Journal of Robotics Research, vol. 31, no. 1, pp. $3-23,2012$.

[12] K. Tahara, S. Arimoto, and M. Yoshida, "Dynamic object manipulation using a virtual frame by a triple soft-fingered robotic hand," in IEEE International Conference on Robotics and Automation (ICRA), 2010, pp. 4322-4327.

[13] A. Kawamura, K. Tahara, R. Kurazume, and T. Hasegawa, "Dynamic grasping of an arbitrary polyhedral object," Robotica, vol. 31, no. 4 pp. 511-523, 2013.

[14] W. Shaw-Cortez, D. Oetomo, C. Manzie, and P. Choong, "Tactilebased blind grasping: Trajectory tracking and disturbance rejection for in-hand manipulation of unknown objects," American Control Conference (ACC), pp. 693-698, 2019.

[15] S. Ueki, H. Kawasaki, and T. Mouri, "Adaptive control of multifingered robot hand using quaternion," in 17th IFAC World Congress, 2008, pp. 6757-6762.

[16] S. Ueki, H. Kawasaki, T. Mouri, and A. Kaneshige, "Object manipulation based on robust and adaptive control by hemispherical soft fingertips," 18th IFAC World Congress, vol. 18, pp. 14654-14659, 2011

[17] C. Cheah, H. Han, S. Kawamura, and S. Arimoto, "Grasping and position control for multi-fingered robot hands with uncertain jacobian matrices," in IEEE International Conference on Robotics and Automation (ICRA), vol. 3, 1998, pp. 2403-2408.

[18] W. Shaw-Cortez, D. Oetomo, C. Manzie, and P. Choong, "Tactilebased blind grasping: A discrete-time object manipulation controller for robotic hands," IEEE Robotics and Automation Letters, vol. 3, no. 2, 2018

[19] P. Fungtammasan and T. Watanabe, "Grasp input optimization taking contact position and object information uncertainties into consideration," IEEE Transactions on Robotics, vol. 28, no. 5, pp. 1170-1177, 2012

[20] R. Murray, Z. Li, and S. Sastry, A mathematical introduction to robotic manipulation. CRC Press: Boca Raton, FL, USA, 1994.

[21] K. Hang, M. Li, J. Stork, Y. Bekiroglu, F. Pokorny, A. Billard, and D. Kragic, "Hierarchical fingertip space: A unified framework for grasp planning and in-hand grasp adaptation," IEEE Transactions on Robotics, vol. 32, no. 4, pp. 960-972, 2016

[22] W. Shaw-Cortez, D. Oetomo, C. Manzie, and P. Choong, "Grasp constraint satisfaction for object manipulation using robotic hands," IEEE Conference on Decision and Control (CDC), pp. 415-420, 2018

[23] S. P. Bhat and D. S. Bernstein, "A topological obstruction to continuous global stabilization of rotational motion and the unwinding phenomenon," Systems \& Control Letters, vol. 39, no. 1, pp. 63-70, 2000.

[24] E. Lavretsky and K. A. Wise, Robust adaptive control. Springer, 2013.

[25] M. Nahon and J. Angeles, "Real-time force optimization in parallel kinematic chains under inequality constraints," IEEE Transactions on Robotics and Automation, vol. 8, no. 4, pp. 439-450, 1992.

[26] A. Bressan and B. Piccoli, Introduction to the mathematical theory of control. American institute of mathematical sciences Springfield, 2007, vol. 1.

[27] R. Ortega, A. Loria, P. J. Nicklasson, and H. Sira-Ramirez, PassivityBased Control of Euler-Lagrange Systems: Mechanical, Electrical, and Electromechanical Applications, ser. Communications and Control Engineering. Springer, 1998.

[28] H. K. Khalil, Nonlinear Systems. Upper Saddle River, N.J. : Prentice Hall, c2002., 2002. 